\title{
Spiral computed tomography and operative staging of gastric carcinoma: a comparison with histopathological staging
}

\author{
J Davies, A G Chalmers, H M Sue-Ling, J May, G V Miller, I G Martin, D Johnston
}

\begin{abstract}
Background-Much controversy exists as to the value of computed tomography (CT) in the preoperative staging of gastric cancer, because of its limited ability to identify correctly lymph node (LN) metastases, invasion of adjacent organs, or hepatic and peritoneal metastases. Spiral CT scanners have a number of potential advantages over conventional scanners, including the absence of respiratory misregistration, image reconstruction smaller than scan collimation permitting overlapping slices and optimisation of intravenous contrast enhancement.
\end{abstract}

Aim-To compare the performance of spiral CT and operative assessment against formal (TNM) pathological staging.

Patients and methods-A study of 105 consecutive patients who underwent both spiral CT and operative staging was performed. All CT scans were reviewed by a radiologist who commented on tumour location and size, evidence of adjacent organ invasion, lymph node metastases to both $\mathrm{N} 1$ and $\mathrm{N} 2$ nodes, and evidence of hepatic and peritoneal metastases. All patients underwent careful operative assessment at the time of surgery, along the lines suggested by Rohde and colleagues.

Results-Spiral CT remained poor at identifying LN metastases to both $\mathrm{N} 1$ and N2 lymph nodes, with sensitivity ranging from 24 to $43 \%$; specificity, however, was $100 \%$. Operative staging was superior, with sensitivities between 84 and $94 \%$, but specificity was much lower (63-74\%). Spiral CT correctly detected 13 of 17 cases of invasion of either the colon or the mesocolon (sensitivity $76 \%$ ) compared with 16 of 17 cases at operative staging (sensitivity 94\%). Spiral CT correctly identified three of six cases with invasion of the pancreas (sensitivity $50 \%$ ) compared with six of six cases on operative staging (sensitivity $100 \%$ ). Spiral CT correctly identified 12 of 17 cases of peritoneal metastases (sensitivity $71 \%$ ) and four of seven cases of hepatic metastases (sensitivity $57 \%$ ). Conclusion-Whilst spiral CT remains poor at identifying lymph node metastases, it correctly identified most cases with invasion of either the colon or the mesocolon and half the cases of pancreatic invasion. It was of value in detecting peritoneal metastases and some cases with hepatic metastases. At present, at Leeds General Infirmary spiral CT is performed routinely on all patients with gastric cancer and a selective staging laparoscopy policy is adopted in those patients in whom the status of the peritoneal cavity and liver is in doubt.

(Gut 1997; 41: 314-319)

Keywords: gastric carcinoma; spiral computed tomography; operative staging

Although the incidence of gastric cancer is decreasing worldwide, it remains the fourth commonest cause of death from cancer in the West. ${ }^{1}$ Increased awareness among the public and medical profession, and open access endoscopy have facilitated the detection of some lesions early in the clinical course, ${ }^{23}$ although most patients still present with advanced disease. ${ }^{4}$ At present, surgery is the only therapeutic option offering a chance of cure. Despite increased referral for consideration of surgical treatment, $15-30 \%$ of patients have unresectable disease and undergo no procedure other than open biopsy. ${ }^{4-6}$

Traditionally, staging has been performed at the time of surgery, although a sensitive and reliable staging tool which could identify patients with unresectable disease would be of great benefit in saving patients from unnecessary laparotomy. Computed tomography (CT) remains the most widely used imaging modality for preoperative staging ${ }^{5}$ but its value remains controversial. After some initial encouraging reports advocating its routine use ${ }^{7-9}$ the more recent literature is far less enthusiastic, ${ }^{10-13}$ reporting limited ability to identify correctly lymph node metastases, invasion of adjacent organs or hepatic and peritoneal metastases. For these reasons, many believe that CT is too insensitive for it to be of any value in routine staging. ${ }^{10-13}$

More recently, other staging investigations have been developed, notably endoluminal ultrasound (EUS), which has been shown to be superior to CT in the assessment both of depth of invasion through the stomach wall (T-stage) and of N1 and N2 lymph nodes (N-stage). ${ }^{14-16}$ Endoluminal ultrasound does, however, have limitations, as it is available only in specialist centres and requires considerable expertise to perform and interpret. Additionally, EUS is unable to assess fully the liver and is of limited value in the assessment of peritoneal disease. Formal staging laparoscopy has been
Mr J Davies. 2 May 1997 
advocated, ${ }^{17}{ }^{18}$ though not as a routine investigation for all patients with gastric cancer.

In the past few years spiral or helical CT technology has been developed which has a number of potential advantages over conventional systems. ${ }^{19-21}$ Spiral CT permits the acquisition of a volume data set from which axial slices are reconstructed. The table on which the patient lies moves at a constant speed while the $x$ ray tube produces a sustained exposure. Respiratory misregistration is eliminated as the scan acquires data during a single breath-hold. Scan reconstruction at intervals smaller than scan collimation produces overlapping slices, which improves the detection of small lesions. In addition, contrast enhancement can be optimised due to a shorter scanning time which, along with the development of high heat capacity tubes, allows images to be acquired in the phase of contrast enhancement most appropiate to the pathology being assessed. For example, arterial phase imaging, portal venous phase imaging or a combination of the two are now possbile. ${ }^{19-21}$ With these potential advantages it is hoped that spiral CT may improve the staging of gastric cancer, although at present there is little in the literature concerning the use of this new technology. ${ }^{22}$ The aim of this study was to compare the performance of spiral CT and operative assessment against formal (TNM) pathological staging. ${ }^{23}$

\section{Patients and Methods}

Between 31 October 1993 and 28 February 1996 we studied 105 consecutive patients who underwent both preoperative spiral CT and operative assessment. Each patient had a preoperative histological diagnosis of gastric adenocarcinoma established on the basis of upper gastrointestinal endoscopic biopsy. The median age was 69 years (range 33-92 years). Sixty eight patients were male and 37 female.

The CT examinations were performed on a Philips Tomoscan SR 7000 scanner (120 Kvp and 225-300 mAs) and all but four patients (96\%) underwent contrast enhanced spiral CT within two weeks of operation. Thirty minutes before scanning, after an overnight fast, patients were asked to drink $500 \mathrm{ml}$ of $3 \%$ Urografin 370 (Schering Health Care Ltd, West Sussex, UK) to opacify the small bowel and stomach. A minority of patients $(n=20)$ were also given effervescent Carbex granules and solution (Ferrings Pharmaceuticals, Middlesex, UK) and $10 \mathrm{mg}$ intravenous Buscopan (Boehringer Ingelheim Ltd, Bracknell, UK) to produce a distended gas-filled stomach. Intravenous contrast Ultravist $300(150 \mathrm{ml})$ (Schering Health Care) was delivered by power injector, at a rate of $3 \mathrm{ml}$ per second, through a cannula in the antecubital fossa. All patients were scanned in the supine position. Initially, a volume acquisition of the thorax was performed using $10 \mathrm{~mm}$ slice thickness, a reconstruction index of $10 \mathrm{~mm}$ and a table speed of $10 \mathrm{~mm}$ per second. A second volume acquisition of the abdomen from the dome of the diaphragm to the pelvic brim was performed using $10 \mathrm{~mm}$ slice thickness with a reconstruction index of $5 \mathrm{~mm}$. This meant that slices were acquired every $10 \mathrm{~mm}$, but reconstructed at $5 \mathrm{~mm}$ intervals, which produced overlapping slices. The second volume was timed to begin liver imaging $60-70$ seconds after the onset of contrast injection.

The radiologist was unaware of both the operative and histological findings. In a minority of cases $(n=16)$ hard copy images were not available and the images were retrieved and reviewed on the diagnostic console. The proforma included questions concerning scan quality (gastric distension and diagnostic quality) and specific questions regarding tumour location and size, evidence of adjacent organ invasion, lymph node metastases to both N1 and N2 nodes, and evidence of hepatic and peritoneal metastases. For the purposes of this study, nodes were considered to be involved if they were greater than $1 \mathrm{~cm}$ in diameter. ${ }^{914} 24$ Adjacent organs were considered to be involved if there was CT evidence of direct spread of the tumour into adjacent organs. Specific areas of fat plane effacement were also compared with adjacent evidence of fat plane preservation.

Most patients underwent potentially curative radical D2 gastrectomy $(\mathrm{n}=74),{ }^{25} 22$ underwent palliative resection and the remaining nine patients underwent palliative bypass $(n=3)$, open/close laparotomy $(n=3)$ or laparoscopy only $(n=3)$. All patients underwent operative assessment, at the time of surgery, along the lines suggested by Rohde and colleagues. ${ }^{26}$ This included assessment of tumour size, location, adjacent organ invasion, lymph node metastases, and evidence of hepatic and peritoneal disease. In patients in whom only palliative resection was possible, we still attempted to remove as much tumour tissue as possible. In the course of the 105 operations, "en bloc" splenectomy $(n=16)$, distal pancreatectomy $(n=9)$ and transverse colectomy $(n=16)$ were performed.

In this study, spiral CT assessment and operative assessment were compared with the final TNM histopathological stage. ${ }^{23}$ Individual lymph node groups were dissected out and placed in separate specimen pots by the surgeon who performed the operation. The gastrectomy specimen and associated lymph node groups were transferred to the pathology department. The stomach was opened whilst still fresh along the greater curve and pinned to a cork board before fixation. Lymph nodes were dissected individually from the surrounding fatty tissue and each gland assessed histologically. The depth of invasion of the primary tumour was meticulously defined. N1 nodes were defined as lymph nodes within $3 \mathrm{~cm}$ of the primary tumour. N2 lymph nodes were defined as those more than $3 \mathrm{~cm}$ from the primary tumour, including nodes located along the left gastric, common hepatic, splenic or coeliac arteries. ${ }^{23}$

The results are expressed as sensitivity and specificity, positive predictive value (PPV) and negative predictive value (NPV). 


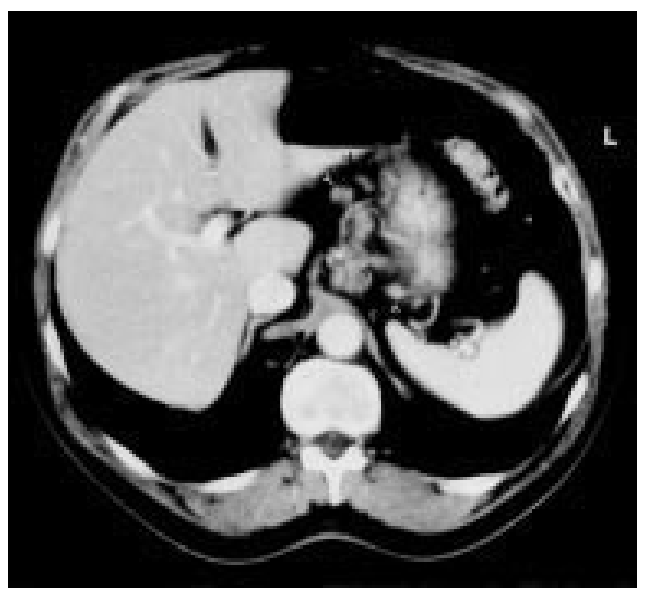

Figure 1: Axial section through the upper abdomen showing multiple gastro-hepatic ligament lymph nodes.

Sensitivity is defined as the number of true positive cases divided by the sum of the true positive cases plus the false negative cases.

Specificity is the number of true negative cases divided by the sum of the true negative cases plus the false positive cases.

Positive predictive value is the number of true positive cases divided by the sum of the true positive cases plus the false positive cases.

Negative predictive value is the number of true negative cases divided by the sum of the true negative cases plus the false negative cases.

\section{Results}

SCAN QUALITY

Gastric distension was considered adequate in $90(86 \%)$ of the 105 patients assessed. The scan was considered to be of either adequate or good diagnostic quality in $97(92 \%)$ of the 105 patients.

TUMOUR LOCATION

Spiral CT correctly identified the location of the primary tumour in $63(66 \%)$ of the 96 patients who underwent gastric resection. Spi-

TABLE 1 Spiral CT and operative status of both the N1 and N2 lymph nodes in 94 patients with gastric cancer when compared with final histology

\begin{tabular}{llllll}
\hline & \multirow{2}{l}{ N1 lymph nodes } & & \multicolumn{2}{l}{ N2 lymph nodes } \\
\cline { 2 - 3 } \cline { 5 - 6 } & Spiral CT & Operative & & Spiral CT & Operative \\
\hline Sensitivity & $15 / 62(24)$ & $58 / 62(94)$ & & $19 / 44(43)$ & $37 / 44(84)$ \\
Specificity & $32 / 32(100)$ & $20 / 32(63)$ & & $50 / 50(100)$ & $37 / 50(74)$ \\
PPV & $15 / 15(100)$ & $58 / 70(82)$ & & $19 / 19(100)$ & $37 / 50(74)$ \\
NPV & $32 / 79(40)$ & $20 / 24(83)$ & & $50 / 75(67)$ & $37 / 44(84)$ \\
Accuracy & $47 / 94(50)$ & $78 / 94(83)$ & & $69 / 94(73)$ & $74 / 94(79)$ \\
\hline
\end{tabular}

Results are expressed as number (\%).

Assessment of lymph node metastases was performed in only 94 of the 96 patients who underwent resection as in two cases there were inadequate numbers of both $\mathrm{N} 1$ and $\mathrm{N} 2$ lymph nodes.

TABLE 2 Spiral CT and operative assessment of both colonic/mesocolonic and pancreatic invasion in patients with gastric cancer when compared with final histology

\begin{tabular}{|c|c|c|c|c|}
\hline \multirow[b]{3}{*}{ Sensitivity } & \multicolumn{2}{|c|}{ Colonic/mesocolonic invasion } & \multicolumn{2}{|c|}{ Pancreatic invasion } \\
\hline & Spiral CT & Operative & Spiral CT & Operative \\
\hline & $13 / 17 \quad(76)$ & $16 / 17$ & $(50)$ & $6 / 6 \quad(100)$ \\
\hline Specificity & $84 / 88 \quad(95)$ & $84 / 88$ & $88 / 89(99)$ & $87 / 89(98)$ \\
\hline PPV & $13 / 17 \quad(76)$ & $16 / 20$ & $3 / 4 \quad(75)$ & $6 / 8 \quad(75)$ \\
\hline NPV & $84 / 88 \quad(95)$ & $84 / 85$ & $88 / 91$ (97) & $87 / 87(100)$ \\
\hline Accuracy & $97 / 105(92)$ & $100 / 105(95)$ & $91 / 95(96)$ & $93 / 95(98)$ \\
\hline
\end{tabular}

Results are expressed as number (\%). ral CT ascribed the location of the primary tumour to the incorrect position in eight patients, in four of whom gastric distension was considered to be inadequate. In 25 patients, the primary tumour could not be visualised on CT: of these cases, 13 (52\%) were early gastric cancers. ${ }^{25}$ Operative staging correctly identified the tumour location in $93(97 \%)$ of the 96 patients who underwent resection, but in three cases the surgeon was unable to locate the primary tumour at operation (all were early gastric cancers). ${ }^{25}$

\section{LYMPHADENOPATHY}

N1 nodes

Spiral CT correctly identified involvement of $\mathrm{N} 1$ lymph nodes in 15 of 62 patients, giving a sensitivity of only $24 \%$ (fig 1 ). However, CT correctly identified all 32 patients who did not have nodal involvement (specificity 100\%) (table 1). Operative assessment of N1 nodal status was much superior to that of spiral CT, correctly identifying 58 of the 62 cases (sensitivity 94\%), although there was a number of false positives (that is, nodes deemed to be involved at operation that subsequently proved to be reactive), giving a specificity of $63 \%$ (20/ 32).

\section{N2 nodes}

Spiral CT correctly identified involvement of $\mathrm{N} 2$ nodes in 19 of 44 cases, giving a sensitivity of $43 \%$ and a specificity of $100 \%$ (table 1 ). Operative assessment of $\mathrm{N} 2$ nodal involvement was similar to that of N1 nodes, correctly identifying 37 of 44 cases (sensitivity $84 \%$ ), although overstaging of $\mathrm{N} 2$ nodal involvement remained a problem (specificity $37 / 50$ or $74 \%$ ).

INVASION OF ADJACENT ORGANS

Colon and mesocolon

Seventeen patients had direct invasion of either the colon or the mesocolon at histological assessment of the resected specimen. Spiral CT correctly detected such invasion in 13 of the 17 patients (sensitivity $76 \%$ ), but on four occasions spiral CT provided a false positive result (specificity 95\%) (table 2). Surgical staging correctly identified 16 of the 17 patients with direct invasion (sensitivity $94 \%$ ) with a similar proportion of false positives (specificity $95 \%$ ).

\section{Pancreas}

Only six patients had evidence of invasion of the pancreas on histological examination and spiral CT correctly identified this in three cases (sensitivity 50\%) (fig 2). On one occasion, spiral CT suggested pancreatic invasion when subsequent histological examination revealed only inflammatory change, giving a specificity of $99 \%$ (88/89) (table 2). Operative assessment identified all six cases with pancreatic invasion (sensitivity $100 \%$ ), and on two occasions suggested invasion which was subsequently refuted on histological examination.

\section{DISTANT METASTASES}

Spiral CT correctly identified the presence of liver metastases in four of the seven patients in whom metastases were seen at the time of sur- 


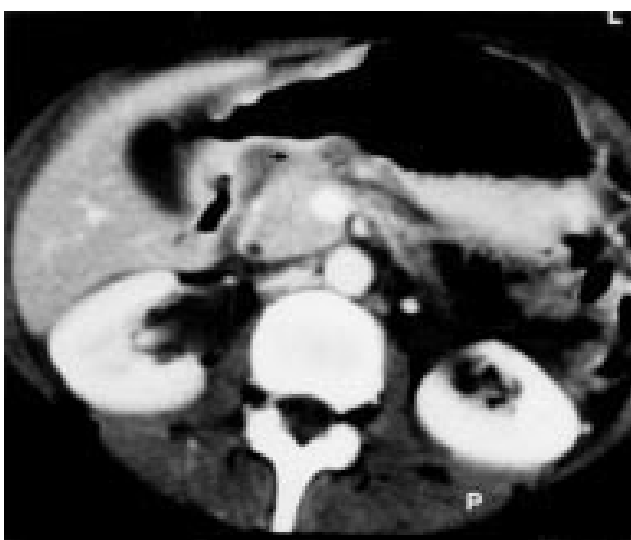

Figure 2: Axial section showing circumferential antral tumour with invasion of the pancreatic neck.

TABLE 3 Spiral CT assessment of both peritoneal and hepatic metastases in 105 patients with gastric cancer when compared with laparotomy/histology findings

\begin{tabular}{lllll}
\hline & \multicolumn{2}{l}{ Peritoneal metastases } & \multicolumn{2}{l}{ Hepatic metastases } \\
\hline Sensitivity & $12 / 17$ & $(71)$ & $4 / 7$ & $(57)$ \\
Specificity & $82 / 88$ & $(93)$ & $98 / 98$ & $(100)$ \\
PPV & $12 / 18$ & $(67)$ & $4 / 4$ & $(100)$ \\
NPV & $82 / 87$ & $(94)$ & $98 / 101$ & $(97)$ \\
Accuracy & $94 / 105(89)$ & $102 / 105$ & $(97)$ \\
\hline
\end{tabular}

Results are expressed as number (\%).

gery, and confirmed histologically on needle biopsy (sensitivity 57\%), but missed three others, two of which were small (less than $10 \mathrm{~mm}$ ) subscapular metastases. However, spiral CT had a specificity of $100 \%$ in detection of liver metastases, as there were no false positive results (table 3 ).

Peritoneal disease, discontinuous from the resected specimen, was found at laparotomy and confirmed histologically in 17 cases. This was correctly identified in 12 of 17 cases on spiral CT (sensitivity $71 \%$ ) (fig 3) although peritoneal metastases were suggested on spiral CT and not found at operation in six cases (specificity of 93\%) (table 3).

\section{Discussion}

Spiral CT is a very inaccurate method of identifying lymph node metastases from gastric carcinoma. Our findings are in keeping with those of other authors 812141627 who have reported sensitivities ranging from 48 to $91 \%$.

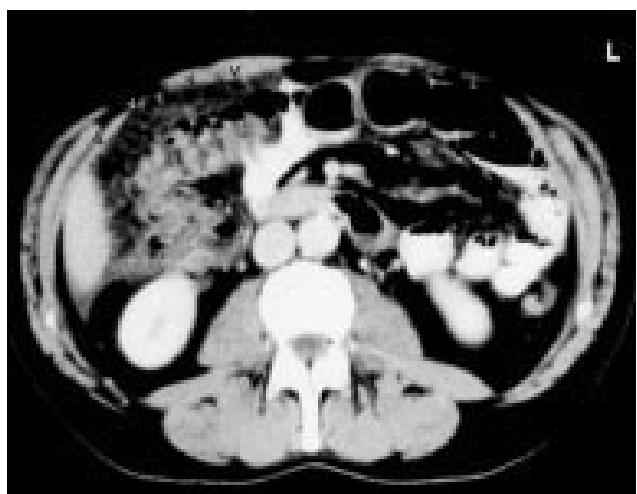

Figure 3: Axial section through the tip of the liver showing extensive peritoneal deposits anterior to the hepatic flexure. Also a trace of ascites is noted within the subhepatic space.
Detection of involved N1 lymph nodes was particularly poor, with a sensitivity of only $24 \%$. Spiral CT was highly specific, however, in the detection of uninvolved N1 nodes (specificity of $100 \%)$. In the report from Andaker et $a l,{ }^{13} \mathrm{CT}$ yielded results similar to ours, with a sensitivity of $26 \%$ and specificity of $99 \%$ when perigastric nodes were examined. The reason that sensitivity is so low and specificity so high is probably partly because of the large cut-off size $(10 \mathrm{~mm})$ used to define "involved" nodes. In other series, a smaller size was used than the $10 \mathrm{~mm}$ used in our series and any lowering of the size criterion will obviously permit identification of a greater proportion of malignant nodes, thus increasing sensitivity but at a cost of lowered specificity. ${ }^{27} 28$

The ability of spiral CT to identify involvement of N2 lymph nodes was better than for $\mathrm{N} 1$ nodes, the sensitivity being $48 \%$ and specificity, $100 \%$. Sendler et a ${ }^{18}$ have reported that CT is better at demonstrating involved N2 lymph nodes than involved N1 nodes for a number of reasons: differentiating between involved nodes near the tumour and the primary tumour itself can be difficult, nodes of normal size may contain metastatic deposits, and enlarged N2 lymph nodes are more clearly distinguished from adjacent, highly enhancing vessels using the early phase of dynamic scanning. ${ }^{12} 1828$

Operative assessment was a more sensitive method of assessment than spiral CT at identifying involved $\mathrm{N} 1$ and $\mathrm{N} 2$ nodes (sensitivity 84-94\%). Increased sensitivity, however, was associated with lower specificity (63-74\%). Operative assessment relies not only on the size, but also on the texture of lymph nodes. There is little information in the world literature concerning the relative accuracy of operative staging compared with histological staging. However, in one of the few studies of the accuracy of operative staging ${ }^{29}$ surgeons underestimated cases with lymph node involvement, but overestimated invasion of adjacent organs. Our results are in agreement with the findings of Madden et $a l,{ }^{30}$ who examined surgical staging in 78 consecutive patients with gastric cancer, and found that surgeons tended to overestimate lymph node involvement. Most recently, EUS has been shown to be more accurate than either CT or operative assessment in detecting lymph node metastases from cancer of the upper gastrointestinal tract. ${ }^{15} 1631$ This is probably because EUS does not only use the size of lymph nodes to identify lymph node metastases but also takes account both of the shape and of the internal echo pattern of the nodes. EUS poses its own problems, however, as it requires greater expertise in interpretation and is limited to viewing nodes that are located no more than $6-7 \mathrm{~cm}$ from the gastric lumen. $^{32}$

There is at present only one other reported study in which spiral CT was used to assess lymph node involvement in gastric cancer, and that was in a Japanese population with predominantly early gastric cancers. ${ }^{22}$ Fukuya et al found that the incidence of positive lymph nodes varied with size $(5 \%$ for nodes smaller 
than $5 \mathrm{~mm}, 23 \%$ for nodes between $10-14 \mathrm{~mm}$ and $84 \%$ for nodes greater than $14 \mathrm{~mm}$ in size). It seems from this study that spiral CT is superior to conventional CT in the detection of nodal metastases.

Preoperative knowledge of adjacent organ invasion and distant metastases is important in planning the operative procedure, allowing the surgeon to decide whether surgery is likely to be potentially curative or palliative in nature and also whether additional organs may have to be resected. Compared with previous reports of conventional CT, spiral CT scanning seems to be both a sensitive and specific method for demonstrating invasion of both the mesocolon and colon. Spiral CT correctly identified 13 of the 17 cases (sensitivity of $76 \%$ ) with invasion of the colon and mesocolon. In contrast, Andaker et $a l^{13}$ found conventional CT to be insensitive in evaluating invasion of the colon and mesocolon, demonstrating this in only three of 12 cases with histological evidence of invasion (sensitivity 25\%). Operative assessment was superior to spiral CT, with a sensitivity of $94 \%$ and specificity of $95 \%$.

The pancreas is an organ that has been notoriously difficult to assess both at laparotomy and on preoperative imaging. Previous reports have described sensitivities for assessment of pancreatic invasion varying from 27 to $95 \% .{ }^{12}$ In our hands, spiral CT remains poor at demonstrating invasion of the pancreas. Only half the patients with pancreatic invasion were correctly identified on spiral CT, whereas surgical assessment correctly identified all six patients. Both methods were highly specific, CT with one false positive and operative assessment with two false positive results. CT visualisation of the pancreas can be difficult in patients with gastric cancer as they are frequently malnourished and visceral fat between the organs is depleted. ${ }^{33}$ Although a clear fat plane between stomach and pancreas can reliably be taken to indicate non-invasion, the absence of a fat plane between these organs does not necessarily imply invasion. In similar fashion, irregularity of the interface does not necessarily represent invasion. ${ }^{34}$ Differentiation between inflammatory adhesion, oedema and true invasion, both on spiral CT and at operation, can be extremely difficult. ${ }^{12}$ In this study, both false positive operative assessments were found to represent inflammatory reaction on subsequent histological examination. All patients were scanned in a supine position, but it has been suggested that prone scanning improves the ability of CT to identify pancreatic invasion. Perhaps in the future EUS may be able to provide more accurate information about pancreatic invasion. ${ }^{14-16}$

Spiral CT has the potential to demonstrate both liver and peritoneal metastases because it can be used to examine the entire abdominal cavity, unlike EUS.$^{18}$ Spiral CT correctly demonstrated a surprisingly low proportion of the cases with liver metastases (four of seven cases; sensitivity $57 \%$ ). These metastases were identified at operation and confirmed histologically. In two of the three cases missed, however, the metastases were single, small (less than
$10 \mathrm{~mm}$ ) and within a single lobe. Small metastases (less than $10 \mathrm{~mm}$ ) are typical of those found in patients with gastric cancer ${ }^{18}$ and the results presented are very much in keeping with those of other series. It is important to remember that for assessment of hepatic metastases the CT findings were compared against the operative findings confirmed histologically. However, there may well have been other small intrahepatic metastases which were not identified at operative assessment which means there could have been a number of false negative cases which were not identified. Ideally intra-operative ultasound would have been used as a gold standard for detecting hepatic metastases. ${ }^{18}$

It has been reported previously that CT is limited in the demonstration of peritoneal metastases, being able to identify peritoneal disease only in the presence of ascites. ${ }^{18}$ Whilst CT can identify obvious gross peritoneal disease, with large omental cake-like deposits, it is poor at visualising small individual peritoneal nodules due to its limits of resolution. Surprisingly, spiral CT correctly demonstrated 12 of the 18 cases in which peritoneal metastases were found at sites separate from the main tumour, thus it seems that spiral CT may be superior to older scanners in demonstrating peritoneal disease.

It comes as no surprise that $\mathrm{CT}$ is poor at predicting the stage of disease in patients with gastric cancer. Although CT is able to demonstrate the stomach wall as a three layer structure, corresponding to the mucosal, submucosal and musculo-serosal layers, the outer musculo-serosal layer is thin in places and cannot always be visualised. ${ }^{35}$ Destruction of this layered structure together with gastric wall thickening may be seen on scanning, although correct assignment to a $\mathrm{T}$ stage (T1-T3) may be extremely difficult or even impossible. This, together with limited ability of CT to identify lymph node metastases, means that this modality is of limited value as a predictor of overall stage of gastric cancer. From this study, however, it is clear that due to high specificity, when CT demonstrates an "involved" node, it is highly likely to contain tumour on histological examination.

Of most concern from recent reports is the suggestion that CT can over-stage patients, by falsely predicting the presence of irresectable disease which was not confirmed at subsequent laparotomy. In this series, spiral CT was found to be highly specific, which meant that there was a low incidence of false positive findings. For surgeons who practise radical "D2" resections, the most important information required is whether distant metastatic (M1) disease is present, which implies that a potentially curative resection cannot be performed.

In conclusion, we found that spiral CT remained poor at identifying lymph node metastases and in determining depth of tumour invasion (T1-T3), but was highly specific which meant that a positive spiral CT result could be relied upon. It was also of value in identifying invasion of adjacent organs and some cases with distant metastatic (M1) 
disease. At present, we perform spiral CT routinely on all patients and adopt a selective staging laparoscopy policy in those patients in whom the status of the peritoneal cavity and liver remains in doubt. Whether the introduction of EUS will change our practice remains to be seen.

Part of this work was presented at the 5th United European Gastroenterology Week in Paris, 2-6 November 1996, and has been published in abstract form (Gut 1996; 39 (suppl 3): A38)

1 Coleman MP, Esteve J, Damiecki P, Arslan A, Renard H. Trends in cancer incidence and mortality. IARC Sci Publ 1993; 12: 193-228.

2 Hallissey MT, Allum WH, Jewkes AJ, Ellis DJ, Fielding JWL. Early detection of gastric cancer. BMF 1990; 301: JWL. Eart

3 Sue-Ling HM, Martin I, Griffith J, Ward DC, Quirke P, Johnston D, et al. Early gastric cancer: 46 pateints treated in one surgical department. Gut 1992; 33: 1318-22.

4 Allum WH, Powell DJ, McConkey CC, Fielding JWL. Gastric cancer: a 25-year review. Br f Surg 1989; 76: 535-40.

5 Wanebo HJ, Kennedy BJ, Chmiel J, Steele G Jr, Winchester D, Osteen R. Cancer of the stomach. A patient care study by the American College of Surgeons. Ann Surg 1993; 218: 583-92.

6 Lundh G, Burn JI, Kolig G, Claude RA, Thomson JWW, Elk PJ, et al. A co-operative international study of gastric cancer (under the auspices of the International Federation of Surgical Colleges). Ann R Coll Surg Engl 1974; 54: 219 28.

7 Moss A, Schnyder P, Marks W, Margulis AR. Gastric adenocarcinoma: a comparison of the accuracy and economics of staging by computed tomography and economics of staging by computed tom

surgery. Gastroenterology 1981; 80: 45-50.
8 Dehn TCB, Reznek RH, Nockler IB, White FE. The pre-operative assessment of advanced gastric cancer by pre-operative assessment of advanced gastric cance
computed tomography. Br F Surg 1984; 71: 413-7.

9 Vallgren S, Hedenbro J, Gotberg S, Walther B. Preoperative computed tomography for evaluation of tumour growth in patients with gastric cancer. Acta Chir Scand 1985; 151 571-3.

10 Fraser I, Nash R, James DC. Computed tomography in gastric cancer. Br f Surg 1985; 72: 249.

11 Cook AO, Levine BA, Sirinek KR, Gaskill HV. Evaluation of gastric adenocarcinoma: Abdominal computed tomography does not replace celiotomy. Arch Surg 1986; 121 603-6.

12 Sussman SK, Halvorsen RA, Illescas FF, Cohan RH, Saeed $M$, Silvermen WM, et al. Gastric adenocarcinoma: CT versus surgical staging. Radiology 1988; 167: 335-40.

13 Andaker L, Morales O, Hojer H, Backstrand B, Borch K, Larsson J. Evaluation of pre-operative computed tomLarsson J. Evaluation of pre-operative computed tom-

14 Botet J, Lightdale CJ, Zauber AG, Gerdes H, Winawer SJ, Urmacher C, et al. Preoperative staging of gastric cancer: comparison of endoscopic US and dynamic CT. Radiology 1991; 181: 426-32.

15 Lok Tio T, Coene P, Schouwink MH, Tygat GNJ. Esophagogastric carcinoma: preoperative TNM classification with endosonography. Radiology 1989; 173: 411-7.
Ziegler K, Sanft C, Zimmer T, Zeitz M, Felsenberg D, Stein $\mathrm{H}$, et al. Comparison of computed tomography, en- dosonography, and intra-operative assessment in TNM taging of gastric carcinoma. Gut 1993; 34: 604-10.

17 Watt I, Stewart I, Anderson D, Bell G, Anderson JR. Laparoscopy, ultrasound and computed tomography in cancer of the oesophagus and gastric cardia: A prospective comparison for detecting intra-abdominal metastases. $\mathrm{Br} \mathcal{F}$ Surg 1989; 76: 1036-9.

18 Sendler A, Dittler HJ, Feussner H, Nekarda H, Bollschweiler E, Fink U, et al. Preoperative staging of gastric cancer as precondition for multimodal treatment. World 7 Surg 1995; 19: 501-8.

19 Zeman RK, Fox SH, Silverman PM, Davros WJ, Carter LM, Griego D, et al. Helical (spiral) CT of the abdomen. AfR Am f Roentgenol 1993; 160: 719-25.

20 Herts BR, Einstein DM, Paushter DM. Spiral CT of the abdomen: artifacts and potential pitfalls. AfR Am f Roentgenol 1993; 161: 1185-90.

21 Heiken JP, Brink JA, Vannier MW. Spiral (helical) CT. Radiology 1993; 189: 647-56.

22 Fukuya T, Honda H, Hayashi T, Kaneko K, Tatashi Y, Ro T, et al. Lymph-node metastases: efficacy of detection with helical CT in patients with gastric cancer. Radiology 1995; 197: 705-11.

23 Herman P, Sobbing LH. TNM classification of malignant tumours. Berlin: Springer Verlag, 1987: 43-6.

24 Maruyama M, Baba M. Gastric carcinoma: radiology of the upper gas

25 Sue-Ling HM, Johnston D, Martin IG, Dixon MF, Lansdown MRJ, McMahon MJM, et al. Gastric cancer: a Lansdown MRJ, McMahon MJM, et al. Gastric canc

26 Rohde H, Rau E, Koster R, Gebbensleben B, Stutzer H. A surgeon's checklist for a standardized staging procedure in patients with cancer of the stomach. Scand $\mathcal{F}$ Gastroenterol 1987; 22 (suppl 133): 36-9.

27 Triller J, Roder R, Stafford A, Schroder R. CT in advanced gastric cancer: Is exploratory laparotomy avoidable? Eur $\mathcal{F}$ Radiol 1986; 6: 181-6.

28 Cho JS, Kim JK, Rho SM, Lee HY, Jeong HY, Lee CS. Preoperative assessment of gastric carcinoma: value of twophase dynamic CT with mechanical IV injection of contrast material. AfR Am f Roentgenol 1994; 163: 69-75.

29 Rohde H, Koster R, Gebbensleben B, Stutzer H, Rau E. Errors of surgical staging in patients with cancer of the stomach. Scand $\mathcal{F}$ Gastroenterol 1987; 22 (suppl 133): 27-30.

30 Madden MV, Price SK, Learmonth GM, Dent DM. Surgical staging of gastric carcinoma: sources and consequences of error. Br f Surg 1987; 74: 119-21.

31 Greenberg J, Durkin M, Van Drunnen M, Aranha GV Computed tomography or endoscopic ultrasonography in preoperative staging of gastric or esophageal tumors. Surgery 1994; 116: 696-702.

32 Dittler HJ, Siewert JR. Role of endoscopic ultrasonography in gastric carcinoma. Endoscopy 1993; 25: 162-6.

33 Kleinhaus U, Militianu D. Computed tomography in the preoperative evaluation of gastric carcinoma. Gastrointest Radiol 1988; 13: 97-101.

34 Tsuburaya A, Noguchi Y, Matsumoto A, Kobayashi S, Masukawa K, Horiguchi K. A pre-operative assessment of adjacent organ invasion by stomach carcinoma with high resolution computed tomography. Surg Today 1994; 24: 299-304.

35 Minami M, Kawauchi N, Itai Y, Niki T, Sasaki Y. Gastric tumors: radiologic-pathologic correlation and accuracy of T staging with dynamic CT. Radiology 1992; 185: 173-8. 